\title{
Autonomic dysfunction in primary Sjogren's syn- drome: a prospective cohort analysis of 154 Korean patients
}

Jung Hee Koh, Seung-Ki Kwok, Jennifer Lee, and Sung-Hwan Park

Division of Rheumatology, Department of Internal Medicine, College of Medicine, Seoul St. Mary's Hospital, The Catholic University of Korea, Seoul, Korea

Received: July 13, 2015

Revised : August 4, 2015

Accepted: August 10, 2015

\section{Correspondence to}

Sung-Hwan Park, M.D.

Division of Rheumatology, Department of Internal Medicine, College of Medicine, Seoul St. Mary's Hospital, The Catholic University of Korea, 222 Banpo-daero, Seocho-gu, Seoul o6591, Korea

Tel: +82-2-2258-6011

Fax: +82-2-3476-2274

E-mail: rapark@catholic.ac.kr
Background/Aims: To determine the prevalence of autonomic dysfunction among Korean patients with primary Sjogren's syndrome (pSS) and its associations with the clinical features of pSS.

Methods: We analyzed 154 participants from the Korean Initiative of primary Sjogren's Syndrome (KISS) as a prospective pSS cohort and 154 age- and sexmatched healthy controls. A standardized 5-minute, supine, resting heart rate variability (HRV) test was performed, and autonomic dysfunction was defined as standard deviation of normal-to-normal RR intervals $(\mathrm{SDNN})<30 \mathrm{~ms}$ in patients $<50$ years old and $\mathrm{SDNN}<20 \mathrm{~ms}$ in patients $\geq 50$ years old. The associations between autonomic dysfunction and various clinical features of pSS were analyzed. Results: The overall autonomic activity in patients with pSS was significantly lower than that in controls. Autonomic dysfunction with the HRV test was observed in $35.7 \%$ of the KISS participants and was associated with a higher European League Against Rheumatism Sjogren's Syndrome Patient Reported Index fatigue score $(p=0.024)$. Raynaud's phenomenon was a more frequent clinical presentation in pSS patients with autonomic dysfunction than in those without autonomic dysfunction (29.4\% and $14.4 \%$, respectively; $p=0.048)$. Decreased parasympathetic activity was observed in $41.6 \%$ of pSS patients. No differences were found in the oral and ocular signs of pSS according to the decreased parasympathetic activity.

Conclusions: In Korean patients with pSS, decreased and imbalanced autonomic activity is prevalent and is associated with fatigue. However, an association between autonomic dysfunction and glandular manifestations was not detected.

Keywords: Sjogren's syndrome; Autonomic nervous system; Fatigue; Heart rate variability

\section{INTRODUCTION}

Primary Sjogren's syndrome (pSS) is an autoimmune disease that affects the exocrine glands, leading to the development of keratoconjunctivitis sicca and xerostomia [1]. The prevalence of extraglandular manifesta- tions (EGMs) including cytopenia, arthritis, neuropathy, interstitial lung disease, and renal disease varies from $40 \%$ to $50 \%$ [2] to $86 \%$ [3]. Peripheral neuropathy is a well-known EGM in patients with pSS [4] and presents as a mild sensory, mixed, trigeminal, or latent motor neuropathy and mononeuritis multiplex [5]. Peripher- 
al neuropathy has been reported in approximately $20 \%$ of patients with pSS [4]. The autonomic nervous system (ANS) is part of the peripheral nervous system (PNS) and is responsible for maintaining homeostasis in the body. Several studies have described ANS dysfunction in pSS $[4,6-8]$.

Autonomic dysfunction may affect both glandular manifestations and EGMs in patients with pSS. Since the degree of exocrine gland destruction and exocrine function are poorly correlated, a possible mechanism involving disturbed neural stimulation of the residual glands regulates secretion has been proposed [9]. In addition, some studies have demonstrated that autonomic dysfunction correlates with pSS-associated symptoms and disease activity $[7,10]$.

The ANS can be assessed using various methods. Short-term heart rate variability (HRV) is the most commonly used, fastest, and non-invasive method for objective measurement of autonomic function. It analyzes the variability of time interval between $\mathrm{R}$ waves [11]. Several studies have demonstrated an association between autonomic dysfunction and pSS using questionnaires on autonomic symptoms $[8,10]$ or tests for dynamic orthostasis $[4,6,7]$. A comprehensive analysis encompassing physiological variables, including assessment of autonomic dysfunction using HRV in pSS patients, has not been fully investigated.

In this study, we evaluated the autonomic dysfunction in a large cohort of Korean patients with clinically well-characterized pSS compared to healthy controls by using HRV. Then, we determined the prevalence of autonomic dysfunction in patients with pSS. In addition, we explored the relationships among autonomic dysfunction and disease activity, patient-reported outcome measures, as well as other potentially relevant clinical and laboratory variables.

\section{METHODS}

\section{Study population}

All pSS patients in this study were participants of the Korean Initiative of primary Sjogren's Syndrome (KISS). KISS was formed in 2013 with the aim of establishing a nationwide prospective cohort with the overall clinical data and samples of pSS patients and developing diag- nostic and treatment tools for pSS. Informed consent was obtained from all patients according to the principles of the Declaration of Helsinki. This study was approved by the Institutional Review Board of Seoul St. Mary's Hospital (KC13ONMIo646). All data were collected and managed with the Clinical Research and Trial Management System (iCReaT; Korea National Institutes of Health, Korea Centers for Disease Control and Prevention).

The recruitment began in the Seoul St. Mary's Hospital, a tertiary care university hospital and referral center in Seoul, Korea, in October 2013. By April 2015, the database included $175 \mathrm{pSS}$ patients from the Seoul St. Mary's hospital, who fulfilled the 2002 American-European Consensus group classification criteria [12] and/or the 2012 American College of Rheumatology criteria [13]. The exclusion criteria were a history of radiation to the head and neck areas, chronic hepatitis C or human immunodeficiency virus infections, previous lymphoproliferative disease, sarcoidosis, graft-versus-host disease, amyloidosis, immunoglobulin G4-related disease, and associated systemic autoimmune diseases such as systemic lupus erythematosus, rheumatoid arthritis, mixed connective tissue disease, primary biliary cirrhosis, vasculitis, autoimmune hepatitis, and systemic sclerosis. One male patient, three with diabetes mellitus four with hypertension and 13 with fibromyalgia were not included in this study, because gender, diabetes mellitus, hypertension, and fibromyalgia can affect the HRV test results $[14,15]$. Fig. 1 shows the number of participants involved in this study from assessment for eligibility. Finally, 154 patients from the KISS cohort were included in this study. Each pSS patient was age-matched to a healthy control who had visited our hospital for a regular check-up. None of the patients had concomitant conditions such as neuropsychiatric disease, amyloidosis, renal failure, diabetes mellitus, hypertension, cardiac arrhythmia, and hypo/hyperthyroidism.

\section{Assessment of autonomic function}

Participants were asked to refrain from eating and drinking caffeinated beverages for at least 8 hours before the examination. A standardized 5-minute supine resting study, including a spectral analysis of the heart rate, was conducted. Data collection and HRV analysis were performed using the SA-300oP (Medicore Co., 

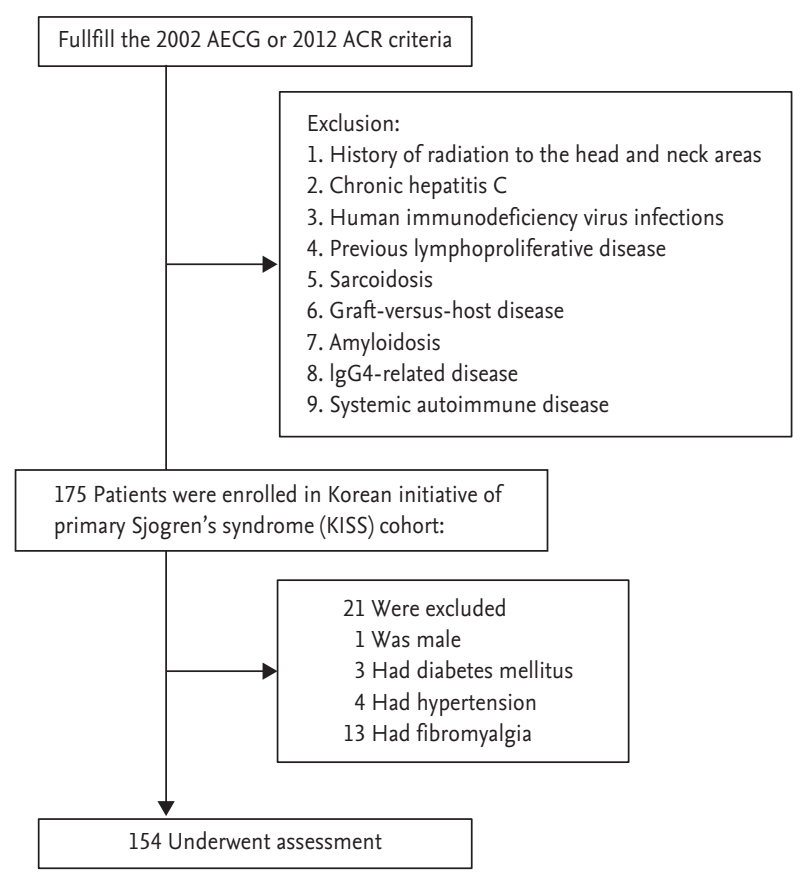

Figure 1. Enrollment, and patients selection $[12,13]$. AECG, American-European Consensus Group; ACR, American College of Rheumatology; IgG4, immunoglobulin $\mathrm{G}_{4}$.

Seongnam, Korea). In all pSS patients and controls, the following HRV parameters were analyzed [11].

(1) Time domain

Standard deviation of normal-to-normal RR intervals (SDNN, ms)

Root mean square of successive differences in normal to normal intervals (RMSSD, ms)

(2) Frequency domain

Total power (T'P) component (o to $0.4 \mathrm{~Hz}$ )

Low frequency (LF) component $(0.04$ to $0.15 \mathrm{~Hz}$ )

High frequency (HF) component ( 0.15 to $0.4 \mathrm{~Hz}$ )

LF/HF ratio

LF or HF in normalized units (n.u.): LF or HF/(TP very $L F) \times 100$

According to the low TP, the raw HF and LF could be relatively low. Normalized LF and HF components would therefore better demonstrate the behavior of the sympathetic and parasympathetic branches of the ANS. Autonomic dysfunction was defined as a SDNN $<30 \mathrm{~ms}$ in patients $<50$ years old and $\mathrm{SDNN}<20 \mathrm{~ms}$ in patients $\geq 50$ years old, based on the large Korean short-term HRV study and age-related influences $[16,17]$.

The 75th percentile of the LF/HF ratio in healthy con- trols was 1.6 and was considered as an upper reference limit. In this study, the higher LF/HF ratio indicated relatively decreased parasympathetic activity.

\section{Assessment of other clinical features of pSS}

The following data were collected for all KISS participants: European League Against Rheumatism (EULAR) Sjogren's Syndrome Disease Activity Index (ESSDAI; range, o to 123) [18], EULAR Sjogren's Syndrome Patient Reported Index (ESSPRI; range, o to 10) [19], Patient's global assessment, Physician's global assessment, unstimulated salivary flow rate, xerostomia inventory (range, 11 to 55) [20], ocular surface disease index (OSDI; range, o to 100) [21], ocular stain score (OSS), tear film break-up time (TBUT), and meibomian gland dysfunction grade.

\section{Statistical analysis}

All data were analyzed using SAS software version $9 \cdot 3$ (SAS Institute, Cary, NC, USA). The Shapiro-Wilk test was used to test for departures from normality of distribution for continuous outcome variables. Variables were usually skewed; continuous variables are therefore expressed as median (interquartile range [IQR]), while categorical data are expressed in absolute and percentage values (\%). When comparing groups, the Mann-Whitney U test was applied to two groups of continuous variables, and the chi-square test was performed for categorical variables. Statistical significance was set at $p<0.05$.

\section{RESULTS}

\section{Baseline characteristics}

The participants consisted of 154 women with a median age of 55 years (IQR, 47 to 60) and a median pSS duration of 2.1 years (IQR, 0.5 to 4.8). All participants fulfilled the 2002 classification criteria [12] $(\mathrm{n}=151,98.1 \%)$ and/or the 2012 classification criteria [13] $(\mathrm{n}=114,74.0 \%)$. All participants were non-smokers, and 66.2\% $(\mathrm{n}=102)$ were postmenopausal.

Current EGMs were observed in $73.6 \%$ of the participants. Autonomic dysfunction was observed with the HRV test in $35.7 \%$ of the participants. The autoimmune antibody profiles revealed anti-Ro/SSA (anti-Ro/ 
Table 1. Heart rate variability parameters of patients with primary Sjogren's syndrome and controls

\begin{tabular}{|c|c|c|c|}
\hline HRV domain & Patients with pSS $(\mathrm{n}=154)$ & Controls $(n=154)$ & $p$ value \\
\hline Age, yr & $55(47-60)$ & $55(47-60)$ & 0.623 \\
\hline Body mass index, $\mathrm{kg} / \mathrm{m}^{2}$ & $22.5(20.7-24.2)$ & $22.0(20.4-24.2)$ & 0.262 \\
\hline Heart rate, $/ \mathrm{min}$ & $68(63-74)$ & $71(64-77)$ & 0.004 \\
\hline SDNN, ms & $26.3(21.6-34.2)$ & $35.0(25.9-57.3)$ & $<0.001$ \\
\hline RMSSD, ms & $18.8(14.0-30.0)$ & $21.3(14.2-31.1)$ & 0.345 \\
\hline Total power, $\mathrm{ms}^{2}$ & $463.4(298.2-793.2)$ & $588.1(288.4-1,259.8)$ & 0.042 \\
\hline Low frequency, ms ${ }^{2}$ & $104.2(56.2-181.5)$ & $119.9(54 \cdot 3-312.4)$ & 0.035 \\
\hline Low frequency, n.u. & $55 \cdot 3(55 \cdot 3-68.8)$ & $48.3(38.0-60.6)$ & 0.096 \\
\hline High frequency, ms ${ }^{2}$ & $77.6(39.8-162.7)$ & $123.5(55.1-326.0)$ & 0.001 \\
\hline High frequency, n.u. & $43.2(29.6-64.0)$ & $51.5(38.8-61.1)$ & 0.027 \\
\hline $\mathrm{LF} / \mathrm{HF}$ ratio & $1.3(0.5-2.2)$ & $0.9(0.6-1.6)$ & 0.042 \\
\hline
\end{tabular}

Values are presented as median (interquartile range).

HRV, heart rate variability; pSS, primary Sjogren's syndrome; SDNN, standard deviation of normal-to-normal RR intervals; RMSSD, root mean square of successive difference in normal to normal intervals; n.u., normalized unit; LF, low frequency; HF, high frequency.

Sjogren's syndrome A antibody) in 90.3\% and anti-La/ SSB (anti-La/Sjogren's syndrome B antibody) in $46.8 \%$ of the participants. The median ESSDAI and ESSPRI scores were 3 (IQR, o to 6) and 5 (IQR, 4 to 6), respectively.

\section{Autonomic dysfunction in patients with pSS}

The overall autonomic influence (SDNN and RMSSD) was lower in pSS patients $\geq 50$ years old than in those $<50$ years old, whereas the parasympathetic and sympathetic activity (LF, [n.u.], HF, [n.u.], and LF/HF ratio) was not different according to age (Supplementary Table 1). We compared the HRV indices between age- and sex-matched healthy controls and patients with pSS. SDNN and TP, the parameters related to the overall autonomic activity, were significantly lower in patients with PSS than in controls during the 5-minute supine resting study (Table 1). The normalized HF component was significantly low in patients with pSS compared to healthy controls $(p=0.027)$, whereas the normalized LF component was not significantly different between patients with pSS and healthy controls. The LF/HF ratio was higher in patients with pSS than in healthy controls (1.3 and 0.9, respectively, $p=0.042$ ). Since the HF component is mainly modulated by the parasympathetic system, and the LF component is regulated by both the sympathetic and parasympathetic drive, a reduced normalized HF component and not significantly elevated normalized LF component indicated a relative impair- ment of the parasympathetic system. The higher LF/HF ratio reflects relatively dominant sympathetic activity. In the circumstance with a decreased normalized HF component and not elevated normalized LF component, the higher ratio indicated relatively decreased parasympathetic activity.

\section{Comparison of clinical manifestations between pSS patients with and without autonomic dysfunction}

Age, disease duration, and body mass index were not associated with autonomic dysfunction. Raynaud's phenomenon was observed in $29.4 \%$ of patients with autonomic dysfunction, whereas it was observed in $14.4 \%$ of patients without autonomic dysfunction $(p=0.048)$. EGMs defined as the presence of disease activity in any ESSDAI domain except the glandular domain alone were not different between patients with and without autonomic dysfunction. A comparison of the glandular symptom severity indices assessed using the OSDI and xerostomia inventory, ocular signs assessed using the OSS, meibomian gland dysfunction and TBUT, and unstimulated salivary flow rate for 15 minutes in patients with and without autonomic dysfunction revealed no significant differences.

The overall ESSDAI and ESSPRI scores were not different according to the presence of autonomic dysfunction. However, the ESSPRI fatigue score was higher in patients with autonomic dysfunction (6 [5 to 7$]$ vs. 5 [3 
Table 2. Comparison of clinical manifestations between patients with and without autonomic dysfunction in primary Sjogren's syndrome

\begin{tabular}{|c|c|c|c|c|}
\hline Characteristic & Total & $\begin{array}{l}\text { Autonomic dysfunction } \\
\qquad(\mathrm{n}=55)\end{array}$ & $\begin{array}{l}\text { No autonomic dysfunction } \\
\qquad(\mathrm{n}=99)\end{array}$ & $p$ value \\
\hline Age, yr & $55(47-60)$ & $52(46-59)$ & $55(46-59)$ & 0.355 \\
\hline Disease duration, yr & $2.1(0.5-4.8)$ & $1.2(0.2-4.0)$ & $2.3(0.6-5.8)$ & 0.122 \\
\hline Body mass index, $\mathrm{kg} / \mathrm{m}^{2}$ & $23(21-24)$ & $22(21-25)$ & $23(21-24)$ & 0.677 \\
\hline Menopause & $102(66.2)$ & $34(61.8)$ & $68(68.7)$ & 0.477 \\
\hline Current EGMs & $109 / 148(73.6)$ & $38 / 51(74 \cdot 5)$ & $71 / 97(73.2)$ & $>0.999$ \\
\hline Raynaud's phenomenon & $29 / 148(19.6)$ & $15 / 51(29.4)$ & $14 / 97(14.4)$ & 0.048 \\
\hline \multicolumn{5}{|l|}{ Serologic profile } \\
\hline Anti-Ro/SSA positive & $139(90.3)$ & $50(90.9)$ & $89(89.9)$ & $>0.999$ \\
\hline Anti-La/SSB positive & $72(46.8)$ & $27(49.1)$ & $45(45 \cdot 5)$ & 0.737 \\
\hline \multicolumn{5}{|l|}{ Glandular manifestations } \\
\hline OSDI & $38(18-53)$ & $40(25-60)$ & $34(16-53)$ & 0.210 \\
\hline OSS & $4(2-6)$ & $4(2-6)$ & $4(2-6)$ & 0.299 \\
\hline Xerostomia inventory & $37(30-43)$ & $37(31-42)$ & $36(30-44)$ & 0.870 \\
\hline $\begin{array}{l}\text { Unstimulated salivary flow rate } \\
\leq 1.5 \mathrm{~mL} / 15 \mathrm{~min}\end{array}$ & $125 / 151(82.8)$ & $48 / 54(88.9)$ & $77 / 97(79.4)$ & 0.179 \\
\hline \multicolumn{5}{|l|}{ Outcome measures } \\
\hline ESSDAI & $3(0-6)$ & $3(0-6)$ & $3(0-6)$ & 0.747 \\
\hline ESSPRI & $5(4-7)$ & $5(5-6)$ & $5(4-7)$ & 0.110 \\
\hline Pain & $3(0-5)$ & $3(0-5)$ & $3(0-5)$ & 0.777 \\
\hline Fatigue & $5(5-7)$ & $6(5-7)$ & $5(3-7)$ & 0.024 \\
\hline Dryness & $7(5-9)$ & $8(6-9)$ & $7(5-9)$ & 0.373 \\
\hline \multicolumn{5}{|l|}{ Medications } \\
\hline Hydroxychloroquine & $105(68.2)$ & $39(70.9)$ & $66(66.7)$ & 0.718 \\
\hline Glucocorticoids & $52(33.8)$ & $20(36.4)$ & $32(32 \cdot 3)$ & 0.722 \\
\hline Pilocarpine & $124(80.5)$ & $45(81.8)$ & $79(79.8)$ & 0.834 \\
\hline
\end{tabular}

Values are presented as median (interquartile range) or number (\%).

EGM, extraglandular manifestation; SSA, Sjogren's syndrome A; SSB, Sjogren's syndrome B; OSDI, ocular surface disease index; OSS, ocular stain score; ESSDAI, European League Against Rheumatism (EULAR) Sjogren's syndrome disease activity index; ESSPRI, EULAR Sjogren's Syndrome Patient Reported Index.

to 7]) than in those without autonomic dysfunction $(p=$ 0.024) (Table 2).

Among patients with pSS, $42 \%(n=64)$ showed decreased parasympathetic activity. The decreased parasympathetic activity was not associated with ocular and oral signs such as the OSS, TBUT, presence of meibomian gland dysfunction, and unstimulated and stimulated salivary flow rate (Table 3). The ESSPRI pain score was higher in patients with decreased parasympathetic activity than in those without. None of the HRV parameters correlated with disease specific measurements or laboratory features.

\section{DISCUSSION}

In the present study, we found an overall decrease and imbalance in autonomic activity in patients with pSS compared to healthy controls. This study demonstrated that autonomic dysfunction in pSS was associated with overall fatigue, whereas autonomic dysfunction was poorly associated with exocrine manifestations and EGMs of pSS.

Although the association of autonomic dysfunction with pSS has been debated in the literature $[4,6,7,10,22,23]$, majority of the studies have endorsed a positive associa- 
Table 3. Comparison of clinical manifestations between patients with and without decreased parasympathetic activity in primary Sjogren's syndrome

\begin{tabular}{|c|c|c|c|}
\hline Characteristic & $\begin{array}{c}\text { Decreased parasympathetic } \\
\text { activity }(n=64)\end{array}$ & $\begin{array}{c}\text { Not decreased parasympathetic } \\
\text { activity }(\mathrm{n}=90)\end{array}$ & $p$ value \\
\hline \multicolumn{4}{|l|}{ Ocular signs } \\
\hline Ocular stain score & $4(2-6)$ & $4(2-6)$ & 0.188 \\
\hline Tear break-up time, sec & $3(2-5)$ & $3(2-5)$ & 0.685 \\
\hline MGD & $49 / 78(62.8)$ & $41 / 58(70.7)$ & 0.365 \\
\hline \multicolumn{4}{|l|}{ Oral signs } \\
\hline Unstimulated salivary flow rate, $\mathrm{mL} / 15 \mathrm{~min}$ & $0.3(0-0.8)$ & $0.3(0-1.0)$ & 0.315 \\
\hline Stimulated salivary flow rate, mL/15 min & $6(3-12)$ & $9(3-18)$ & 0.1 \\
\hline ESSPRI & $5(5-7)$ & $5(4-6)$ & 0.363 \\
\hline Pain & $4(1-6)$ & $3(0-5)$ & 0.031 \\
\hline Fatigue & $5(4-7)$ & $5(5-7)$ & 0.921 \\
\hline Dryness & $8(5-9)$ & $7(5-9)$ & 0.872 \\
\hline
\end{tabular}

Values are presented as median (interquartile range) or number (\%).

MGD, meibomian gland dysfunction; ESSPRI, EULAR Sjogren's Syndrome Patient Reported Index.

tion between them. Our results also revealed that many pSS patients have restricted HRV but normal heart rate and blood pressure in the resting state. This discrepancy in the results could be explained by methodological variations in the tests used, such as the conventional cardiovascular reflex test, baroreflex sensitivity test, and HRV test. Because of better sensitivity and reproducibility compared to reflex tests [24], HRV analysis is widely used in ANS investigations. Reduced HRV is a powerful and independent predictor of a cardiac event in the general population [25-27]. Furthermore, recent studies demonstrated that the cardiovascular risk burden in pSS patients is increased compared to that in the general population $[28,29]$. A long-term follow-up study is needed to explore the relationship between autonomic dysfunction and cardiovascular disease.

Exocrine secretion is controlled by the ANS; it consists of liquid secretion, which is mainly parasympathetic, and protein secretion, which is mainly sympathetically modulated [30]. Antibodies to muscarinic acetylcholine receptors have been demonstrated in patients with pSS; this suggests an association between autonomic and exocrine dysfunctions in pSS [31,32]. Imrich et al. [33] demonstrated that decreased glandular parasympathetic activity was associated with hyposalivation. They also described that hemodynamic parameters evaluated using the tilt table test are not different between patients with pSS and controls. Although we could not demonstrate an association between autonomic dysfunction and tear/salivary secretion, we proposed that decreased parasympathetic activity contributed to autonomic imbalance in patients with pSS. Raynaud's phenomenon, which is caused by sympathetic impairment in the regulation of peripheral blood flow, was seen more frequently in pSS patients with autonomic dysfunction than in those without. Decrease in overall autonomic activity is postulated as the cause. Some studies also found relative impairment of the sympathetic nervous system [7,34].

In this study, autonomic dysfunction was associated with the ESSPRI fatigue score in patients with pSS. These findings are consistent with those of previous studies, which reported that orthostatic dysfunction [7] and higher autonomic dysfunction symptom profile scores $[8,10]$ are prevalent in patients with pSS and are correlated with fatigue. Fatigue is a common, disabling extraglandular symptom of pSS, and the prevalence of clinically significant fatigue is $57 \%$ to $74 \%$ [35]. Fatigue is a self-reported symptom and is involved with multiple physiological and psychological interactions. A negative correlation between the noradrenalin level and general fatigue has been observed in patients with pSS [36], suggesting that autonomic dysfunction could be one of the physiological factors associated with fatigue.

Interestingly, decreased parasympathetic activity was 
associated with higher ESSPRI pain scores. Patients with fibromyalgia have significantly lower HRV and parasympathetic activity compared to healthy controls [37]. Although pSS patients with fibromyalgia were excluded in this study, we supposed that patients with pSS were vulnerable to pain when they presented with decreased parasympathetic activity. As the median duration of pSS in the KISS cohort is relatively short, a follow-up study is needed to ascertain that autonomic dysfunction including a decreased parasympathetic activity predicts development of fibromyalgia.

Although autonomic dysfunction is prevalent in patients with pSS, there is no consensus regarding an appropriate method for assessing it. $\mathrm{Ng}$ et al. [7] reported that the total Composite Autonomic Symptom scale score was independently correlated with the ESSPRI, ESSDAI, Hospital Anxiety and Depression Scale, and mental fatigue score. As the PNS domain in the ESSDAI does not include autonomic symptoms, no statistical association between autonomic dysfunction and the PNS domain in the ESSDAI was identified in this study. Assessment of the ANS using the 5-minute HRV test could be considered as an ancillary method for assessing disease activity in patients with $\mathrm{pSS}$.

A potential limitation of the current study is that we did not evaluate the symptoms of autonomic dysfunction. Detailed autonomic symptoms such as orthostatic intolerance, and vasomotor, secretomotor, and gastrointestinal dysfunction are generally assessed using the Composite Autonomic Symptom Scale or autonomic symptom profile. However, this study assessed autonomic dysfunction using HRV in a relatively large, well-defined pSS cohort. In contrast to the self-reported autonomic symptom questionnaire, the short-term HRV test is relatively insusceptible to the respondents. The short-term HRV test is a non-invasive method to objectively evaluate autonomic dysfunction and has been shown to correlate well with the 24-hour HRV test $[17,38]$. Another potential limitation is that patients with autonomic dysfunction could be misclassified. We divided pSS patients into two groups with and without autonomic dysfunction by age dependent SDNN value. This was used because there are no cut-offs available for clinically relevant autonomic dysfunction. This cutoff value was defined according to previous large population based studies $[16,17]$ and was validated in our healthy control (Supplementary Table 1).

In conclusion, autonomic dysfunction is relatively prevalent in patients with pSS and is associated with fatigue. Parasympathetic activity is relatively decreased in these patients; however, it is not associated with exocrine function.

\section{KEY MESSAGE}

1. Autonomic dysfunction is prevalent in patients with primary Sjogren's syndrome and is assessed using the 5-minute supine resting heart rate variability test.

2. Autonomic dysfunction is associated with the severity of fatigue in patients with primary Sjogren's syndrome. However, no association was detected between autonomic dysfunction and exocrine function in these patients.

\section{Conflict of interest}

No potential conflict of interest relevant to this article was reported.

\section{Acknowledgments}

This study was supported by a grant from the Korean Health Technology R\&D Project, Ministry of Health and Welfare, Republic of Korea (HI13Coo16).

We thank So Young Kim, the research nurse for the KISS cohort, for collecting the clinical data and Sun Jun Kim, the technician who performed the heart rate variability test.

\section{REFERENCES}

1. Fox RI. Sjogren's syndrome. Lancet 2005;366:321-331.

2. Voulgarelis M, Tzioufas AG, Moutsopoulos HM. Mortality in Sjogren's syndrome. Clin Exp Rheumatol 2008;26(5 Suppl 51):S66-S71.

3. Hernandez-Molina G, Michel-Peregrina M, Bermudez-Bermejo P, Sanchez-Guerrero J. Early and late extraglandular manifestations in primary Sjogren's syndrome. Clin Exp Rheumatol 2012;30:455.

4. Barendregt PJ, van den Bent MJ, van Raaij-van den Aarssen VJ, et al. Involvement of the peripheral nervous 
system in primary Sjogren's syndrome. Ann Rheum Dis 2001;60:876-881.

5. Andonopoulos AP, Lagos G, Drosos AA, Moutsopoulos HM. The spectrum of neurological involvement in Sjogren's syndrome. Br J Rheumatol 1990;29:21-23.

6. Mandl T, Wollmer P, Manthorpe R, Jacobsson LT. Autonomic and orthostatic dysfunction in primary Sjogren's syndrome. J Rheumatol 2007;34:1869-1874.

7. Ng WF, Stangroom AJ, Davidson A, Wilton K, Mitchell S, Newton JL. Primary Sjogrens syndrome is associated with impaired autonomic response to orthostasis and sympathetic failure. QJM 2012;105:1191-1199.

8. Mandl T, Hammar O, Theander E, Wollmer P, Ohlsson B. Autonomic nervous dysfunction development in patients with primary Sjogren's syndrome: a follow-up study. Rheumatology (Oxford) 2010;49:1101-1106.

9. Humphreys-Beher MG, Brayer J, Yamachika S, Peck AB, Jonsson R. An alternative perspective to the immune response in autoimmune exocrinopathy: induction of functional quiescence rather than destructive autoaggression. Scand J Immunol 1999;49:7-10.

10. Newton JL, Frith J, Powell D, et al. Autonomic symptoms are common and are associated with overall symptom burden and disease activity in primary Sjogren's syndrome. Ann Rheum Dis 2012;71:1973-1979.

11. Task Force of the European Society of Cardiology and the North American Society of Pacing and Electrophysiology. Heart rate variability: standards of measurement, physiological interpretation and clinical use. Circulation 1996;93:1043-1065.

12. Vitali C, Bombardieri S, Jonsson R, et al. Classification criteria for Sjogren's syndrome: a revised version of the European criteria proposed by the American-European Consensus Group. Ann Rheum Dis 2002;61:554-558.

13. Shiboski SC, Shiboski CH, Criswell L, et al. American College of Rheumatology classification criteria for Sjogren's syndrome: a data-driven, expert consensus approach in the Sjogren's International Collaborative Clinical Alliance cohort. Arthritis Care Res (Hoboken) 2012;64:475-487.

14. Martinez-Martinez LA, Mora T, Vargas A, Fuentes-Iniestra M, Martinez-Lavin M. Sympathetic nervous system dysfunction in fibromyalgia, chronic fatigue syndrome, irritable bowel syndrome, and interstitial cystitis: a review of case-control studies. J Clin Rheumatol 2014;20:146-150.

15. Bonnemeier H, Richardt G, Potratz J, et al. Circadian profile of cardiac autonomic nervous modulation in healthy subjects: differing effects of aging and gender on heart rate variability. J Cardiovasc Electrophysiol 2003;14:791799.

16. Park SB, Lee BC, Jeong KS. Standardized tests of heart rate variability for autonomic function tests in healthy Koreans. Int J Neurosci 2007;117:1707-1717.

17. Voss A, Heitmann A, Schroeder R, Peters A, Perz S. Shortterm heart rate variability: age dependence in healthy subjects. Physiol Meas 2012;33:1289-1311.

18. Seror R, Ravaud P, Bowman SJ, et al. EULAR Sjogren's syndrome disease activity index: development of a consensus systemic disease activity index for primary Sjogren's syndrome. Ann Rheum Dis 2010;69:1103-1109.

19. Seror R, Ravaud P, Mariette X, et al. EULAR Sjogren's Syndrome Patient Reported Index (ESSPRI): development of a consensus patient index for primary Sjogren's syndrome. Ann Rheum Dis 2011;70:968-972.

20. Thomson WM, Chalmers JM, Spencer AJ, Williams SM. The xerostomia inventory: a multi-item approach to measuring dry mouth. Community Dent Health 1999;16:12-17.

21. Schiffman RM, Christianson MD, Jacobsen G, Hirsch JD, Reis BL. Reliability and validity of the ocular surface disease index. Arch Ophthalmol 2000;118:615-621.

22. Kovacs L, Paprika D, Takacs R, et al. Cardiovascular autonomic dysfunction in primary Sjogren's syndrome. Rheumatology (Oxford) 2004;43:95-99.

23. Niemela RK, Hakala M, Huikuri HV, Airaksinen KE. Comprehensive study of autonomic function in a population with primary Sjogren's syndrome: no evidence of autonomic involvement. J Rheumatol 2003;30:74-79.

24. Kleiger RE, Bigger JT, Bosner MS, et al. Stability over time of variables measuring heart rate variability in normal subjects. Am J Cardiol 1991;68:626-630.

25. Wulsin LR, Horn PS, Perry JL, Massaro JM, D'Agostino RB. Autonomic imbalance as a predictor of metabolic risks, cardiovascular disease, diabetes, and mortality. J Clin Endocrinol Metab 2015;100:2443-2448.

26. Sajadieh A, Nielsen OW, Rasmussen V, Hein HO, Hansen JF. C-reactive protein, heart rate variability and prognosis in community subjects with no apparent heart disease. J Intern Med 2006;260:377-387.

27. Tsuji H, Larson MG, Venditti FJ Jr, et al. Impact of reduced heart rate variability on risk for cardiac events. The Framingham Heart Study. Circulation 1996;94:2850-2855.

28. Gravani F, Papadaki I, Antypa E, et al. Subclinical atherosclerosis and impaired bone health in patients with 
primary Sjogren's syndrome: prevalence, clinical and laboratory associations. Arthritis Res Ther 2015;17:99.

29. Bartoloni E, Baldini C, Schillaci G, et al. Cardiovascular disease risk burden in primary Sjogren's syndrome: results of a population-based multicentre cohort study. J Intern Med 2015;278:185-192.

30. Konttinen YT, Sorsa T, Hukkanen M, et al. Topology of innervation of labial salivary glands by protein gene product 9.5 and synaptophysin immunoreactive nerves in patients with Sjogren's syndrome. J Rheumatol 1992;19:30-37.

31. Bacman S, Berra A, Sterin-Borda L, Borda E. Muscarinic acetylcholine receptor antibodies as a new marker of dry eye Sjogren syndrome. Invest Ophthalmol Vis Sci 2001;42:321-327.

32. Bacman S, Sterin-Borda L, Camusso JJ, Arana R, Hubscher O, Borda E. Circulating antibodies against rat parotid gland $\mathrm{M}_{3}$ muscarinic receptors in primary Sjogren's syndrome. Clin Exp Immunol 1996;104:454-459.

33. Imrich R, Alevizos I, Bebris L, et al. Predominant glandu- lar cholinergic dysautonomia in patients with primary Sjogren's syndrome. Arthritis Rheumatol 2015;67:13451352.

34. Tumiati B, Perazzoli F, Negro A, Pantaleoni M, Regolisti G. Heart rate variability in patients with Sjogren's syndrome. Clin Rheumatol 2000;19:477-480.

35. Segal B, Thomas W, Rogers T, et al. Prevalence, severity, and predictors of fatigue in subjects with primary Sjogren's syndrome. Arthritis Rheum 2008;59:1780-1787.

36. Barendregt PJ, Visser MR, Smets EM, et al. Fatigue in primary Sjogren's syndrome. Ann Rheum Dis 1998;57:291295.

37. Meeus M, Goubert D, De Backer F, et al. Heart rate variability in patients with fibromyalgia and patients with chronic fatigue syndrome: a systematic review. Semin Arthritis Rheum 2013;43:279-287.

38. Nunan D, Sandercock GR, Brodie DA. A quantitative systematic review of normal values for short-term heart rate variability in healthy adults. Pacing Clin Electrophysiol 2010;33:1407-1417. 


\section{KJIM}

Supplementary Table 1. Comparison of heart rate variability profile according to age stratified by healthy control and pSS patients

\begin{tabular}{|c|c|c|c|}
\hline Variable & $<50$ Years & $\geq 50$ Years & $p$ value \\
\hline \multicolumn{4}{|l|}{ Healthy controls } \\
\hline Heart rate, /min & $73(65-80)$ & $70(64-75)$ & 0.093 \\
\hline $\mathrm{SDNN}, \mathrm{ms}$ & $43.5(34.4-65.2)$ & $32.5(23.4-52.3)$ & 0.001 \\
\hline $\mathrm{RMSSD}, \mathrm{ms}$ & $24.5(20.6-34.9)$ & $17.8(12.7-26.9)$ & $<0.001$ \\
\hline $\mathrm{TP}, \mathrm{ms}^{2}$ & $896.9(551.2-1,586.3)$ & $411.7(202.8-1,029.9)$ & $<0.001$ \\
\hline $\mathrm{LF}, \mathrm{ms}^{2}$ & $202.4(107.1-447 \cdot 3)$ & $89.7(42.0-239.5)$ & $<0.001$ \\
\hline LF, n.u. & $48.7(38.2-60.9)$ & $48.2(37.1-60.6)$ & 0.825 \\
\hline $\mathrm{HF}, \mathrm{ms}^{2}$ & $182.1(123.5-403.9)$ & $74.8(38.6-230.8)$ & $<0.001$ \\
\hline HF, n.u. & $51.3(39.1-61.8)$ & $51.5(38.4-60.2)$ & 0.625 \\
\hline LF/HF ratio & $0.97(0.60-1.58)$ & $0.90(0.66-1.60)$ & 0.773 \\
\hline \multicolumn{4}{|l|}{ pSS patients } \\
\hline Heart rate, /min & $69(65-76)$ & $68(62-73)$ & 0.134 \\
\hline $\mathrm{SDNN}, \mathrm{ms}$ & $31.2(25.2-39.2)$ & $24.4(19.8-32.2)$ & 0.001 \\
\hline $\mathrm{RMSSD}, \mathrm{ms}$ & $25.7(15.6-33.9)$ & $17.1(13.0-24.6)$ & 0.005 \\
\hline $\mathrm{TP}, \mathrm{ms}^{2}$ & $596.8(373.8-899.1)$ & $430.7(270.7-777.7)$ & 0.019 \\
\hline $\mathrm{LF}, \mathrm{ms}^{2}$ & $129.0(70.3-210.4)$ & $85.7(43 \cdot 3-173.6)$ & 0.013 \\
\hline LF, n.u. & $53.2(32.6-65.4)$ & $55.6(35 \cdot 4-71.2)$ & 0.212 \\
\hline $\mathrm{HF}, \mathrm{ms}^{2}$ & $132.0(71.7-222.1)$ & $63.2(33.2-110.0)$ & $<0.001$ \\
\hline HF, n.u. & $43.5(33.3-66.5)$ & $42.9(28.0-59.5)$ & 0.239 \\
\hline LF/HF ratio & $1.25(0.50-1.85)$ & $1.33(0.65-2.49)$ & 0.131 \\
\hline
\end{tabular}

Values are presented as median (interquartile range).

pSS, primary Sjogren's syndrome; SDNN, standard deviation of normal to normal RR intervals; RMSSD, root mean square of standard deviation; TP, total power; LF, low frequency; n.u., normalized unit; HF, high frequency. 